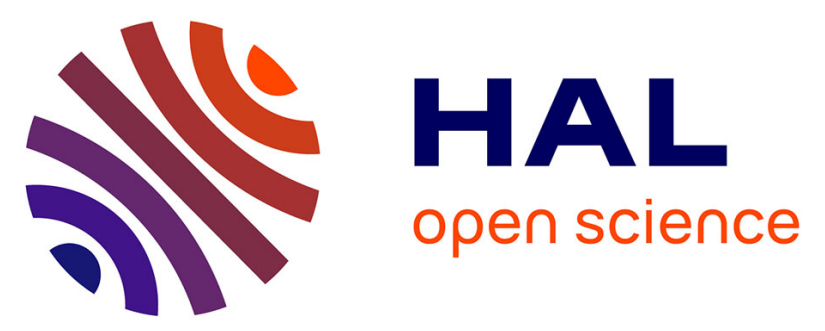

\title{
Calculation of thermal expansion coefficient of glasses based on topological constraint theory
}

Huidan Zeng, F. Ye, Xiaojian Li, L. Wang, Bin Yang, Jie Chen, Xianghua Zhang, L. Sun

\section{- To cite this version:}

Huidan Zeng, F. Ye, Xiaojian Li, L. Wang, Bin Yang, et al.. Calculation of thermal expansion coefficient of glasses based on topological constraint theory. Chemical Physics Letters, 2016, 662, pp.268-272. 10.1016/j.cplett.2016.09.001 . hal-01381137

HAL Id: hal-01381137 https://hal-univ-rennes1.archives-ouvertes.fr/hal-01381137

Submitted on 8 Dec 2016

HAL is a multi-disciplinary open access archive for the deposit and dissemination of scientific research documents, whether they are published or not. The documents may come from teaching and research institutions in France or abroad, or from public or private research centers.
L'archive ouverte pluridisciplinaire HAL, est destinée au dépôt et à la diffusion de documents scientifiques de niveau recherche, publiés ou non, émanant des établissements d'enseignement et de recherche français ou étrangers, des laboratoires publics ou privés. 


\title{
Calculation of Thermal Expansion Coefficient of Glasses Based on
}

\section{Topological Constraint Theory}

Huidan Zeng, ${ }^{1, *}$ Feng Ye, ${ }^{1}$ Xiang Li, ${ }^{1}$ Ling Wang, ${ }^{1}$ Bin Yang, ${ }^{1}$ Jianding Chen, ${ }^{1}$ Xianghua Zhang, ${ }^{2}$ Luyi Sun ${ }^{3}$

${ }^{1}$ Key Laboratory for Ultrafine Materials of Ministry of Education, School of Materials Science and Engineering, East China University of Science and Technology, Shanghai 200237, China

${ }^{2}$ Laboratory of Glasses and Ceramics, Institute of Chemical Science, University of Rennes, 35042, France

${ }^{3}$ Department of Chemical and Biomolecular Engineering and Polymer Program, Institute of Materials Science, University of Connecticut, Storrs, Connecticut 06269, United States

*Corresponding author: Huidan Zeng hdzeng@ecust.edu.cn

\begin{abstract}
In this work, the thermal expansion behavior and the structure configuration evolution of glasses were studied. Degree of freedom based on the topological constraint theory is correlated with configuration evolution; considering the chemical composition and the configuration change, the analytical equation for calculating the thermal expansion coefficient of glasses from degree of freedom was derived. The thermal expansion of typical silicate and chalcogenide glasses was examined by calculating their thermal expansion coefficients (TEC) using the approach stated above. The results showed that this approach was energetically favorable for glass materials and revealed the corresponding underlying essence from viewpoint of configuration entropy. This work establishes a configuration-based methodology to calculate the thermal expansion coefficient of glasses that, lack periodic order.
\end{abstract}

\section{Introduction}

The study of thermal expansion of materials is increasingly significant in both scientific and technological fields [1]. Matching thermal expansion between different materials is a matter of considerable interest for technicians [2]. Scientific researchers have been investigating the nature of thermal expansion behavior of materials for decades [3-7]. However, the essence of glasses' TEC still remains to be explored, due 
to their non-periodic order and complex structures [8-11]. To achieve the prediction of thermal expansion properties of glasses, various early empirical approaches have been explored; for example, the investigation of L. I. Demkina established the equation that describes the relationship between the TEC and each component of glass material [12]. Aside from the empirical approaches, the semi-empirical equation deduced by A. Makishim et al. from the Gruneisen formula is typically used [13]. However, Makishim noted, "Unfortunately, the additive factors vary for each of the authors". Molecular dynamics approaches have been recently developed to interpret the thermal expansion phenomenon especially for crystalline materials [14-16]. The TEC of crystalline materials can be accurately calculated due to their well-defined periodic order. However, the atomic scale modeling of glasses still remains to be a challenge because of their disordered structures. Moreover, the thermodynamic stability of glasses is much larger in terms of time and length required, compared to that of crystals $[17,18]$.

Here we present a model to calculate glasses' TEC based on topological constraint theory (TCT), which is established in previous work by Phillips and Thorpe [19]. Recently, Gupta and Mauro derived the equation that upon introducing the entropy into TCT to calculate the glass transition temperature and the liquid fragility of chalcogenide glasses [20,21]. The difference between oxide and chalcogenide glasses is observed in the case of their bond types, particularly that oxide glasses usually contain ionic bonds. Yue et al. [22,23] and Zeng et al. [24,25] proposed that TCT is also applicable to oxide glasses based on their experimental data, which is also validated in this paper. An illustration of the hardness comes from the study of M. M. Smedskjaer et al, in which it was shown that the hardness of glasses can be readily calculated from its constraint number using TCT [26].

Following a similar strategy, the thermal expansion behavior of chalcogenide glass systems was studied in the work of U. Senapati et al. [27] and L. W. Hyung et al. [28]. It has been observed that the thermal expansion coefficient of a glass material is proportional to its coordination number. Here, a detailed study on different glass-forming units was performed rather than on average sense. The purpose of this work was to unravel the natural connection between thermal expansion and topological constraints by introducing configuration entropy and to derive the equation to calculate the TEC of glasses.

In Section 2, the methodology and the derivation details are described. The calculation of TEC using our approach and the results obtained for the silicate and chalcogenide glasses are presented in Section 3. The summary is subsequently given in Section 4.

\section{Modelling}

A systematic study on thermal expansion behavior, starting from basic thermodynamic formulas to topological constraints calculations, was performed. Glasses have been intensively studied before using various approaches focusing on their micro short and medium-range structures, their macro mechanical, or their 
thermodynamic properties [29,30]. In essence, bond stretching causes the thermal expansion behavior of materials, glass formers relax with increasing the temperature, which leads to the anharmonic vibration of the system [31]. These investigations served as the basic knowledge for comprehension of glasses' isotropy features, as were adopted in this study. This characteristic allowed for the modelling of the TEC from the statistical thermodynamics point of view and provided the prerequisite of the model. The observation that bulk glasses have fixed thermal expansion coefficient values at different directions further support this idea. Models on crystals cannot be directly applied to glasses, but these approaches gave insight into the treatment of glasses. Summarizing this information about glasses, the basic thermodynamic formula was quoted and the linear correlation of TEC and degrees of freedom was finally obtained.

A basic thermodynamic formula describes the relation between internal energy and entropy: $d U=T d S-p d V$, where $T$ is temperature, $S$ is the entropy, $U$ is the internal energy, $p$ is the pressure, and $V$ is the volume. The heat energy absorbed by glasses transforms into two parts: first is the entropy increase, and the rest corresponds to the volume work due to the expansion of glasses. For a homogeneous system in which the internal energy is $U=U(T, V)$ [32], the differential form is given by Eq. (1). It includes the treatment which has the objective to disintegrate the internal energy into two parts, the first part is isochoric heat capacity, and the second part is related to the functions in terms of isothermal compressibility and the volume expansion coefficient. Theoretically, Eq. (1) introduces a method of obtaining the thermodynamic functions related to the volume expansion coefficient.

$$
\begin{aligned}
\mathrm{d} U & =\left(\frac{\partial U}{\partial T}\right)_{V} \mathrm{~d} T+\left(\frac{\partial U}{\partial V}\right)_{T} \mathrm{~d} V=C_{V} \mathrm{~d} T+\left[T\left(\frac{\partial p}{\partial T}\right)_{V}-p\right] \mathrm{d} V \\
& =C_{V} \mathrm{~d} T+\left[-T \frac{a}{K_{T}}-p\right] \mathrm{d} V
\end{aligned}
$$

where $a$ is the volume expansion coefficient, and $K_{T}$ is the isothermal compressibility coefficient. $K_{T}$ is determined by the composition of the materials [33]. It represents the quantity with the same standard compressibility properties, and indicates the thermodynamic stability defined by the second derivative of the changes in volume. With the value of $0 \leq K_{T} \leq \infty$, the thermodynamic equilibrium of a system can be characterized.

$$
a=\frac{1}{V}\left(\frac{\partial V}{\partial T}\right)_{p}, \quad K_{T}=-\frac{1}{V}\left(\frac{\partial V}{\partial p}\right)_{T}
$$

Combining the basic thermodynamic formula with Eq. (2) gives:

$$
T \mathrm{~d} S=C_{V} \mathrm{~d} T-T \frac{a}{K_{T}} \mathrm{~d} V
$$

Gerardo G. Naumis provided an approach to introduce thermodynamics into the topological constraint theory [34]. According to Gerardo G. Naumis, the total entropy 
of a glass material is composed of the floppy modes and the different configurations of the system. Note that the entropy is mainly composed of floppy modes near the softening temperature; this contribution was accordingly calculated as the main entropy. Its contribution to the configurational entropy could be expressed as:

$$
S=N k_{\mathrm{B}} \ln \Omega_{l}{ }^{f}
$$

where $f$ is the degree of freedom, $N$ is the number of atoms, $K_{B}$ is the Boltzmann constant, and $\Omega_{1}$ is the thermodynamic probability. The TEC of the glasses remains slightly changed under its softening temperature. The average thermal expansion coefficient was calculated for engineering applications. The temperature range is $T_{0} \sim$ $T$ (between the room and softening temperature). Integrating the entropy in the range of $T_{0} \sim T$ gives:

$$
\int_{T_{0}}^{T} \mathrm{~d} S=N k_{\mathrm{B}} \ln \Omega_{1} \times \int_{T_{0}}^{T} \mathrm{~d} f .
$$

here $N K_{B} \ln \Omega_{1}=\xi$, so $\mathrm{d} S=\xi \mathrm{d} f$, Eq. (3) can therefore be rewritten as:

$$
\xi \mathrm{d} f=C_{V} \mathrm{~d} \ln T-\frac{a}{K_{T}} \mathrm{~d} V
$$

There is only one independent variable in Eq. (6), since the volume is dependent on temperature; therefore, $\mathrm{d} V=\alpha V \mathrm{~d} T$. So Eq. (6) can be transformed into:

$$
\xi \mathrm{d} f=C_{V} \mathrm{~d} \ln T-\frac{a^{2} V}{K_{T}} \mathrm{~d} T
$$

Using the Gruneisen equation gives:

$$
a=\frac{r C_{V}}{K_{0} V}
$$

where $r$ is the Gruneisen constant, and $K_{0}$ is the bulk elastic modulus. Thermal expansivity is one extremely important thermal parameter, and it is accounted for the Gruneisen's law of thermodynamics [35]; Gruneisen's law of thermodynamics has been expressed by many researchers in various forms such as Mitra and Mishra [36], Tolapadi [37]. Here $r$ and $K_{0}$ are dependent on the material nature. $r=-(\ln v / \ln V)$, where $v$ is the frequency of vibration of the system. Eq. (7) can be rewritten as:

$$
\mathrm{d} f=\frac{a K_{0} V}{\xi r T} \mathrm{~d} T-\frac{a^{2} V}{\xi K_{T}} \mathrm{~d} T
$$

The reference volume is $V_{0}$ at temperature $T_{0}$, so the glass volume $V$ at temperature $T$ is $V=V_{0}+\alpha V_{0}\left(T-T_{0}\right)$, then

$$
\begin{gathered}
\mathrm{d} f=\frac{a K_{0} V_{0}}{\xi r T} \mathrm{~d} T+\frac{a^{2} K_{0} V_{0}}{\xi r} \mathrm{~d} T-\frac{a^{2} K_{0} V_{0} T_{0}}{\xi r T} \mathrm{~d} T \\
-\frac{a^{2} V_{0}}{\xi K_{T}} \mathrm{~d} T-\frac{a^{3} V_{0} T}{\xi K_{T}} \mathrm{~d} T+\frac{a^{3} V_{0} T_{0}}{\xi K_{T}} \mathrm{~d} T
\end{gathered}
$$

Integrating Eq. (10) gives: 


$$
\begin{gathered}
f-f_{0}=\frac{a K_{0} V_{0}}{\xi r} \ln \frac{T}{T_{0}}+\frac{a^{2} K_{0} V_{0}}{\xi r}\left(T-T_{0}\right)-\frac{a^{2} K_{0} V_{0} T_{0}}{\xi r} \ln \frac{T}{T_{0}} \\
-\frac{a^{2} V_{0}}{\xi K_{T}}\left(T-T_{0}\right)-\frac{a^{3} V_{0}}{2 \xi K_{T}}\left(T^{2}-T_{0}^{2}\right)+\frac{a^{3} V_{0} T_{0}}{\xi K_{T}}\left(T-T_{0}\right)
\end{gathered}
$$

Eq. (11) describes the relationship between the degree of freedom and the TEC. The TEC of glasses is of $10^{-6}$ magnitude, so the square term and the cubic term are much smaller than the linear term, which gives:

$$
f \cong f_{0}+a \frac{K_{0} V_{0}}{\xi r} \ln \frac{T}{T_{0}}
$$

$K_{0} V_{0}\left(\ln \left(T / T_{0}\right)\right) / \xi r$ can be treated as constant, since the experiments were carried out under the same measurement conditions. From Eq. (12), the value of $f$ can be calculated if $a$ and $T$ are known. $a$ can be determined from the slope of the linear plot of the thermal expansion along the temperature range of $T_{0}$ to $T$. Here, $f_{0}$ is degree of freedom at temperature $T_{0}$. As can be concluded from Eq. (12), degree of freedom sketchily satisfies the linear relationship with TEC. This equation for calculating TEC from degrees of freedom is outlined in Section 3, and it was applied to both binary silicate and ternary chalcogenide glasses. Comparisons between the calculations and experiments were illustrated with error bars. A detailed description of topological constraint theory for calculating degrees of freedom of glasses is given in the following section.

\section{Calculation}

To calculate TEC of glasses, the value of degree of freedom must be determined beforehand. Topological constraint theory defines two kinds of constraints: linear constraint $(\alpha)$ and angular constraint $(\beta$ and $\gamma)$. For linear and angular constraint, the constraint numbers are $r / 2$ ( $\alpha$ constraints) and $2 r-3$ ( $r \geq 2$, for $\beta$ and $\gamma$ constraint, $r$ is the coordination number), respectively [38]. Further, the total constraints number could be computed with the equation $\sum n_{i} \times[(r / 2)+(2 r-3)]$ (where $n$ is the total number of atoms, $i$ represents the different species of atoms); degree of freedom is thus $f=3-\sum n i \times[(r / 2)+(2 r-3)]$ (3 means three dimensionalities). With the information in Section 2 as background, glasses are generally regarded as between two and three dimensional. Degree of freedom of glasses can thus be determined for a given composition accordingly; a reasonable consideration could be explained according to Mauro et al [20], who suggested that the constraint number is temperature-dependent. For example, the additional angular constraint centered at oxygen (in case of oxide glasses) should be incorporated for the calculation of constraint number at room temperature. Additional constraint at room temperature was not calculated because the thermal expansion behavior happened far away from room temperature for this treatment.

Investigation over the past decades offers abundant data that clarifies the structure features of chalcogenide glasses [39-41]. This information provides their 
atomic structures and helps to construct the structural model with topological constraint theory. Here, for $\mathrm{Ge}_{x} \mathrm{Se}_{1-x}(x=0.15,0.17,0.18,0.2,0.21,0.25 \mathrm{~mol} \%)$ glasses, the structure units mainly consist of $\mathrm{GeSe}_{4}$ tetrahedrons. The selenium behaves like the bridge oxygen in oxide glasses to connect two $\mathrm{GeSe}_{4}$ tetrahedrons together. The coordination numbers of $\mathrm{Ge}$ and Se are 4 and 2, respectively. While the coordination numbers of Ge, As, and Se are 4, 3, and 2 for ternary $\mathrm{Ge}_{x} \mathrm{As}_{y} \mathrm{Se}_{(1-x-y)}(x=0.1, y=0.1,0.2$, $0.25,0.3,0.35,0.4 \mathrm{~mol} \%)$ and binary $\mathrm{As}_{x} \mathrm{Se}_{1-x}(x=0.2,0.3,0.35,0.4,0.425 \mathrm{~mol} \%)$ glasses according to the investigation of M. F. Thorpe [39]. Therefore, degree of freedom was calculated for $\mathrm{Ge}_{x} \mathrm{Se}_{1-x}$ glasses with equation $r / 2$ and $2 r-3$ :

$$
\begin{aligned}
f=3-n(T, x) & =3-\left\{x\left(2 q_{\alpha}(T)+5 q_{\beta}(T)\right)\right. \\
+ & \left.(1-x)\left(\frac{1}{2} q_{\alpha}(T)+q_{\gamma}(T)\right)\right\}
\end{aligned}
$$

where $\alpha$ is the Ge-Se and Se-Se linear constraint, $\beta$ is the Se-Ge-Se, and $\gamma$ is the angular constraint centered at Se. For Ge atoms, the calculation was carried out in units coordinated with $4 \mathrm{Se}$ atoms. Thus, its linear constraint number was $r / 2=2$, and the angular constraint number was $2 r-3=5$. The principles applied here were applicable to the other glasses. For $\mathrm{As}_{x} \mathrm{Se}_{1-x}$ glasses, degrees of freedom can be expressed as:

$$
\begin{aligned}
f=3-n(T, x)= & 3-\left\{x\left(1.5 q_{\alpha}(T)+3 q_{\beta}(T)\right)\right. \\
& \left.+(1-x)\left(\frac{1}{2} q_{\alpha}(T)+q_{\gamma}(T)\right)\right\}
\end{aligned}
$$

where $\alpha$ is the As-Se and Se-Se linear constraint, $\beta$ is the Se-As-Se angular constraint, and $\gamma$ is the angular constraint centered at Se.

Degree of freedom of $\mathrm{Ge}_{x} \mathrm{As}_{y} \mathrm{Se}_{(1-x-y)}$ glasses is:

$$
\begin{aligned}
f=3-n(T, x) & =3-\left\{x\left(2 q_{\alpha}(T)+5 q_{\beta}(T)\right)+y\left(0.75 q_{\alpha}(T)\right.\right. \\
& \left.\left.+3 q_{\gamma}(T)\right)+(1-x-y)\left(\frac{1}{2} q_{\alpha}(T)+q_{\beta}(T)\right)\right\}
\end{aligned}
$$

where $\alpha$ is the Ge-Se, Se-Se and As-Se linear constraint, $\beta$ is the Se-Ge-Se, As-Ge-Se, and Se-As-Se angular constraint, and $\gamma$ is the angular constraint centered at Se.

Good agreement between the calculated and the experimental values of the TEC was achieved for both binary and ternary chalcogenide glasses that spanned a wide range of compositions (FIG. 1). The linear dependence demonstrated for all samples suggests that the linear term in Eq. (12) plays a key role in calculating TEC from degrees of freedom. The linear correlation coefficient $\left(R^{2}\right)$ also validates this with values of $0.9998,0.9910$, and 0.9918 . The slope is 18.1818, 17.6822, 21.5882 for $\mathrm{Ge}_{x} \mathrm{Se}_{1-x}, \mathrm{As}_{x} \mathrm{Se}_{(1-x)}$ and $\mathrm{Ge}_{x} \mathrm{As}_{y} \mathrm{Se}_{(1-x-y)}$ glasses, respectively. Defects in the glasses, such as, miscoordinated atoms or edge-sharing tetrahedron, are a possible reason that deviation occurred between the calculated and actual values [20]. 


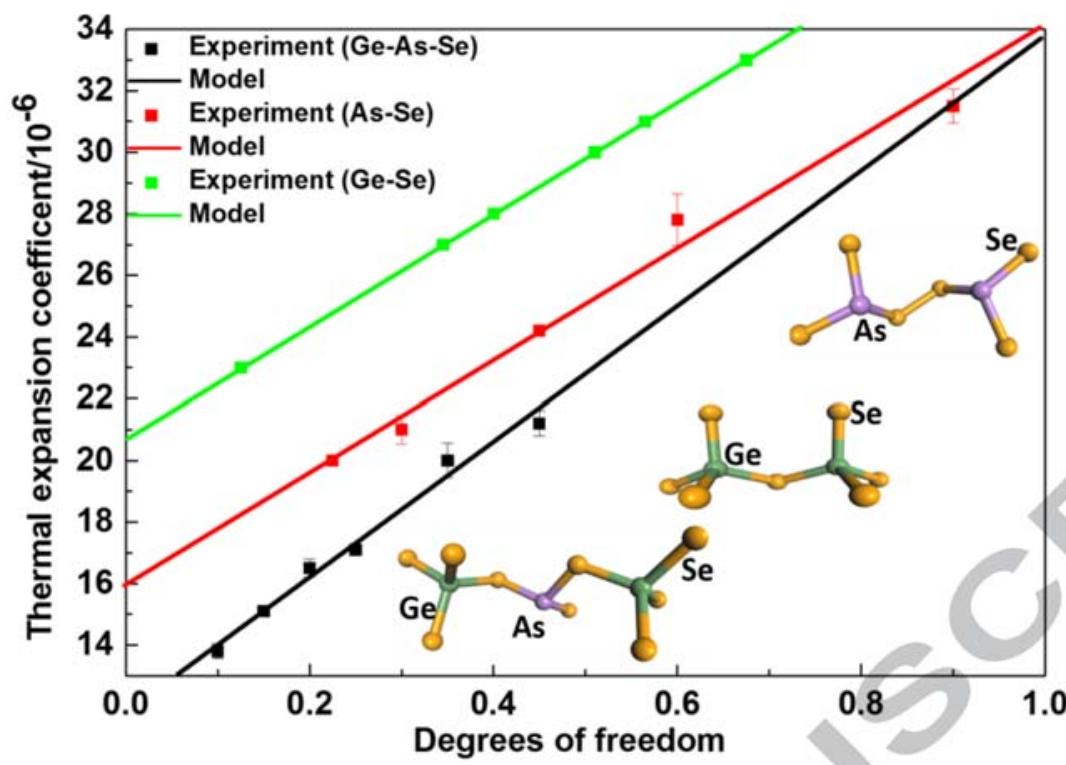

FIG. 1 Experimental TEC as functions of degrees of freedom of $\mathrm{Ge}_{x} \mathrm{Se}_{1-x}, \mathrm{As}_{x} \mathrm{Se}_{1-x}$, and $\mathrm{Ge}_{10} \mathrm{As}_{x} \mathrm{Se}_{(0.9-x)}$ glasses. The square dots represent the experimental results, the solid lines represent the model predictions of the TEC, and the inset shows the coordination structures of the $\mathrm{Ge}_{x} \mathrm{Se}_{1-x}, \mathrm{As}_{x} \mathrm{Se}_{1-x}$, and $\mathrm{Ge}_{10} \mathrm{As}_{x} \mathrm{Se}_{(0.9-x)}$ glasses.

For oxide glasses, the samples of alkali silicate glasses were considered with nuclear magnetic resonance (NMR) data [42] available that helped to construct the structural model and calculate the degree of freedom. Here, silicate glasses containing ionic bonds were again proved to be rationalized by TCT. The constraint strength index, which further extends TCT to overcome its limitations, is introduced here to consider the influence of the alkali ions on glasses. Therefore, degree of freedom of $x \mathrm{Na}_{2} \mathrm{O}-(1-x) \mathrm{SiO}_{2}(x=0.18,0.2,0.25,0.27,0.29,0.32,0.35 \mathrm{~mol} \%), x \mathrm{~K}_{2} \mathrm{O}-(1-x) \mathrm{SiO}_{2}$ $(x=0.2,0.25,0.29,0.33,0.36 \mathrm{~mol} \%)$, and $x \mathrm{Li}_{2} \mathrm{O}-(1-x) \mathrm{SiO}_{2}(x=0.25,0.28,0.25,0.30$, $0.33,0.36,0.40 \mathrm{~mol} \%)$ glasses were calculated with:

$$
\begin{array}{r}
f=3-n(T, x)=3-\{2 B O(x)+N B O(x)+ \\
\left.5\left(\mathrm{Si}^{4}(x)+\mathrm{Si}^{3}(x)+\mathrm{Si}^{2}(x)\right)+q_{\gamma} \times C N \times R_{N B O}^{+}(x)\right\}
\end{array}
$$

where $\mathrm{BO}$ is the bridge oxygen, $\mathrm{NBO}$ is the non-bridge oxygen, $\mathrm{Si}^{\mathrm{n}}$ is the silicon tetrahedron containing $n$ bridge oxygens, $C N$ is the coordination number of the $R^{+}$, and $\gamma$ is the constraints centered at the alkali ions. For $R=\mathrm{Li}, \mathrm{Na}, \mathrm{K}, q_{\gamma}$ (constraint strength index) $=0.75,0.6,0.4$, and $C N=4,5,6$, respectively $[22,23]$. The constraint centered at the modifying cation is linearly related to the charge-to-distance ratio of the modifying cation to the oxygen [43]. The intact $\gamma$ constraints, $q_{\gamma}$ (constraint strength index), can be determined by fitting the experimental transition temperature $\left(T_{g}\right)$ of glasses by using the equation: $T_{g}(x)=\left[3-N_{c}(0)\right] /\left[3-n_{c, \alpha}(x)-n_{c, \beta}(x)-q_{\gamma} \times n_{c, \gamma}(x)\right] \times$ $T_{g}(0)$ (where $N_{c}$ is the reference constraint number, and $n_{c, \alpha}, n_{c, \beta}$, and $n_{c, \gamma}$ are the different constraint number of the composition value of $x$ ).

Good agreement between the calculated results and the experimental data from 
FuXi Gan is displayed in FIG. 2. As parametrized with $q_{\gamma}$ for alkali ions, the incorporation of constraints contributed by glass modifiers like alkali ions in silicate glasses made the calculation more comprehensive compared to its early calculation formalism. Remarkably, it was energetically favorable for this due to the linear correlation coefficients $\left(R^{2}\right)$, which were $0.9959,0.9980$, and 0.9989 (FIG. 2).

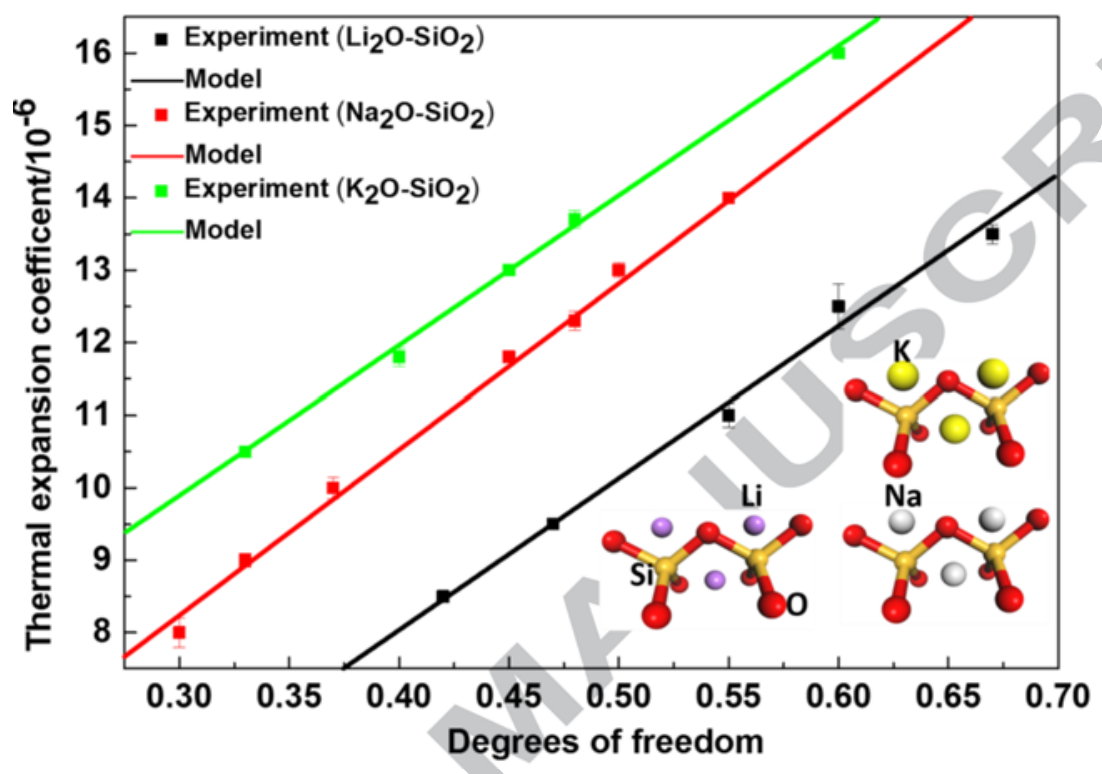

FIG. 2 Experimental TEC as functions of degrees of freedom of $x \mathrm{Na}_{2} \mathrm{O}-(1-x) \mathrm{SiO}_{2}$, $x \mathrm{~K}_{2} \mathrm{O}-(1-x) \mathrm{SiO}_{2}$, and $x \mathrm{Li}_{2} \mathrm{O}-(1-x) \mathrm{SiO}_{2}$ glasses. The square dots represent experimental results, the solid lines represent the predictions of the model. The inset shows the structures of the $\mathrm{Li}_{2} \mathrm{O}-\mathrm{SiO}_{2}, \mathrm{Na}_{2} \mathrm{O}-\mathrm{SiO}_{2}$, and $\mathrm{K}_{2} \mathrm{O}-\mathrm{SiO}_{2}$ glasses.

It is interesting to observe that lithium silicate glasses possessed smaller TEC at the same level of degree of freedom in FIG. 2, even if the constraints centered at Li were $q_{\gamma} \times C N=3$, which is same as in sodium silicate glasses $\left(q_{\gamma} \times C N=3\right.$ for sodium glasses). Although the same constraint number is contributed by the ions of $\mathrm{Li}^{+}$and $\mathrm{Na}^{+}$, the NMR data shows that lithium contributed more to the glass network connectivity compared to the sodium and potassium ions. Increasing the alkali ion content to a relatively high level allowed the $\mathrm{Si}^{4}$ unit content in $0.4 \mathrm{Li}_{2} \mathrm{O}-0.6 \mathrm{SiO}_{2}$ glass to reach up to $17 \mathrm{~mol} \%$ in total silicon coordination polyhedrons, which is obviously larger than $7 \mathrm{~mol} \%$ for both $0.4 \mathrm{Na}_{2} \mathrm{O}-0.6 \mathrm{SiO}_{2}$ and $0.4 \mathrm{~K}_{2} \mathrm{O}-0.6 \mathrm{SiO}_{2}$ glasses [42]. $\mathrm{Si}^{4}$ units possess more constraint number in their medium-range structure compared with $\mathrm{Si}^{3}$ or $\mathrm{Si}^{2}$ since more bridge oxygens connected with. This leads to a small degree of freedom and, consequently, to a relatively small TEC for $\mathrm{Li}_{2} \mathrm{O}-\mathrm{SiO}_{2}$ glasses.

\section{Conclusions}

In this paper, we performed a detailed study on different glass-forming units and unraveled the connection between thermal expansion and topological constraints by 
introducing configuration entropy. The derived equation allows for the computation of TEC of glasses from degree of freedom. As presented in this paper, our approach has two advantages: it does not depend on empirical parameters compared with the early theoretical calculations, and it is a much more time and cost-efficient method compared with molecular dynamic simulations.

Binary silicate and ternary chalcogenide glasses were examined, since they were already intensively studied and structure data was available. The deviation of the calculated and experimental values maybe caused by the defects of the chalcogenide glasses, and this has been of recent research interest. The constraint strength index introduced in the paper for the calculation of degree of freedom for alkali silicate glasses is already verified to overcome the limitations of TCT, which ignored the influence of glass modifiers in early investigations. Results showed that the calculated values were favored in the experimental results, and a strong linear relationship was displayed. It is believed that this work established a configuration-based methodology to calculate the thermal expansion coefficient of materials, like glasses, which lack periodic order.

\section{Acknowledgements}

This work was supported by the National Natural Science Foundation of China (No.21476083, No.51572082) and the Major Program of Science and Technology Commission of Shanghai Municipality (No.ZD14521100604). L. S. acknowledges the partial support from the Key Laboratory for Ultrafine Materials of the Ministry of Education at East China University of Science and Technology. We would like to thank Changjun Peng for useful discussion in this paper.

\section{References}

[1] Monroe J. A., Gehring D., Karaman I., Arroyave R., Brown D. W., Clausen B., Acta. Mater. 102 (2016) 333

[2] Mahato N., Banerjee A., Gupta A., Omar S., Balani K., Prog. Mater. Sci. 72 (2015) 141

[3] M. Blackman, Proc. Phys. Soc., Sec. A (1957)

[4] Bienenstock, A., A.E.R.E., Harwell, Philos. Mag. 9 (1964) 755

[5] Megaw, H.D., Mat. Res. Bull. 6 (1971) 1007

[6] Taylor, D., Mineral. Mag. 38 (1972) 593

[7] Boyer, L.L., Phys. Rev. Lett. 42 (1979) 584

[8] Shelby, J. E., J. Am. Ceram. Soc. 66 (1982) 225

[9] Fabian, J., Allen, P. B., Phys. Rev. Lett. 79 (1997) 1885

[10] Inaba, H., Jpn. J. Appl. Phys. 35 (1996) 4730

[11] A. I. Priven, O. V. Mazurin, Glass Technol. 44 (2002) 156

[12] Demkina, L.I, Glass Ceram.17 (1960) 503

[13] Akio M., J. D. Mackenzie, J. Non-Cryst. Solids 22 (1976) 305 
[14] Gambuzzi, E., Geochim. Cosmochim. Ac.125 (2014) 170

[15] Niranjan, M. K., Kumar, V. S., Karthikeyan, R. J., Phys. D: Appl. Phys. 47 (2014) 285101

[16] Pedesseau L., Ispas S., Kob W., Phys. Rev. B 91 (2015) 134201

[17] Liu Y. M., Jia, Y., Sun Q., Liang E., Phys. Lett. A 379 (2015) 54

[18] Ding, Y., B. Xiao., RSC Adv. 5 (2015) 18391

[19] J.C. Phillips, M.F. Thorpe, Solid State Commun, 53 (1985) 699

[20] Gupta, P. K., J. C. Mauro, J. Chem. Phys. 130 (2009) 094503

[21] Mauro J. C., Gupta, P. K., Loucks, R. J., J. Chem. Phys. 130 (2009) 234503

[22] Hermansen C., Guo X., Youngman R. E., Mauro J. C., Smedskjaer M. M., Yue Y., J. Chem. Phys. 141 (2014) 244502

[23] Hermansen C., Youngman R. E., Wang J., Yue Y., J. Chem. Phys. 142 (2015) 184503

[24] Zeng H., Jiang Q., Liu Z., Li X., Ren J., Chen G., Liu F., Peng S., J. Phys. Chem. B 118 (2015) 5177

[25] Zeng H., Jiang Q., Li X., Ye F., Tian T., Zhang H., Chen G., Appl. Phys. Lett. 106 (2015) 021903

[26] Smedskjaer M. M., Mauro J. C., Yue Y., Phys. Rev. Lett. 105 (2010) 115503

[27] U. Senapati, A. K. Varshneya, J. Non-Cryst. Solids 185 (1995) 289

[28] L. W. Hyung et al., Int. J. Appl. Glass. Sci. (to be published)

[29] I. N. Szabo, Nature 181 (1958) 41

[30] S. R. Elliott, Nature 294 (1981) 24

[31] D. S. Yang, J. M. Lee, J. Phy: Conf. Ser. 430 (2013) 012005

[32] James C. M. Li, J. Chem. Phys. 26 (1957) 909

[33] F. J. Poveda, P. C. M. Castilho, E. D. Mercado, A. R. Fritsch, Phys. Rev. A 92 (2015) 013638

[34] G. G. Naumis, Phys. Rev. E 71 (2005) 026114

[35] G. B. Mitra, A. K. Giri, J. Phy. D: Appl. Phys. 19 (1986) 1065

[36] Mitra. G. B, Mishra. N. K, Br. J. Appl. Phys. 17 (1966) 1319

[37] Tolapadi S, J. Phys. F: Met. Phys. 4 (1974) 42138

[38] J. C. Philips, J. Non-Cryst. Solids 34 (1979) 153

[39] M. F. Thorpe, J. Non-Cryst. Solids 57 (1983) 355

[40] S. Asokan, M. V. N. Prasad, G. Parthasarathy, E. S. R Gopal, Phys. Rev. Lett. 62 (1989) 808

[41] A. Giridhar, S. Mahadevan, A. K. Singh, Bull. Mater. Sci. 8 (1986) 53

[42] H. Maekawa et al., J. Non-Cryst. Solids 127 (1991) 53

[43] Hermansen C., Rodrigues B., P Wondraczek L., Yue Y., J. Chem. Phys., 141 (2014) 244502 


\section{Calculation of Thermal Expansion Coefficient of Glasses Based on}

\section{Topological Constraint Theory}

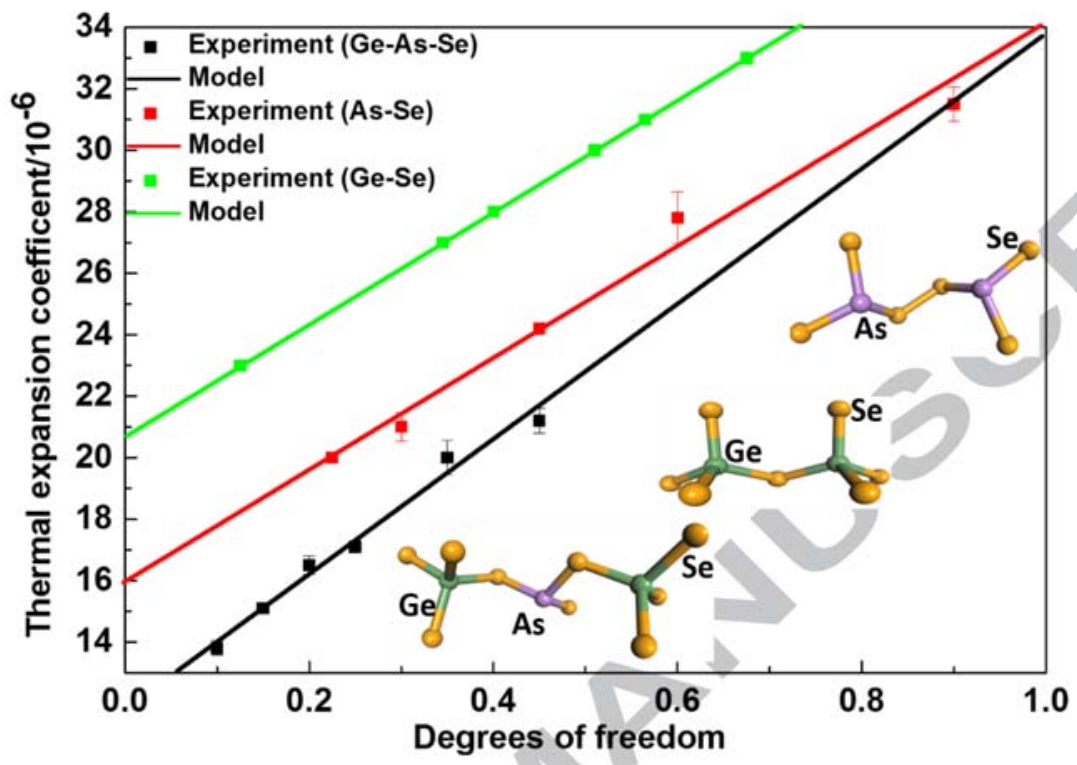

FIG. 1 Experimental TEC as functions of degrees of freedom of $\mathrm{Ge}_{x} \mathrm{Se}_{1-x}, \mathrm{As}_{x} \mathrm{Se}_{1-x}$, and $\mathrm{Ge}_{10} \mathrm{As}_{x} \mathrm{Se}_{(0.9-x)}$ glasses. The square dots represent the experimental results, the solid lines represent the model predictions of the TEC, and the inset shows the coordination structures of the $\mathrm{Ge}_{x} \mathrm{Se}_{1-x}, \mathrm{As}_{x} \mathrm{Se}_{1-x}$, and $\mathrm{Ge}_{10} \mathrm{As}_{x} \mathrm{Se}_{(0.9-x)}$ glasses.

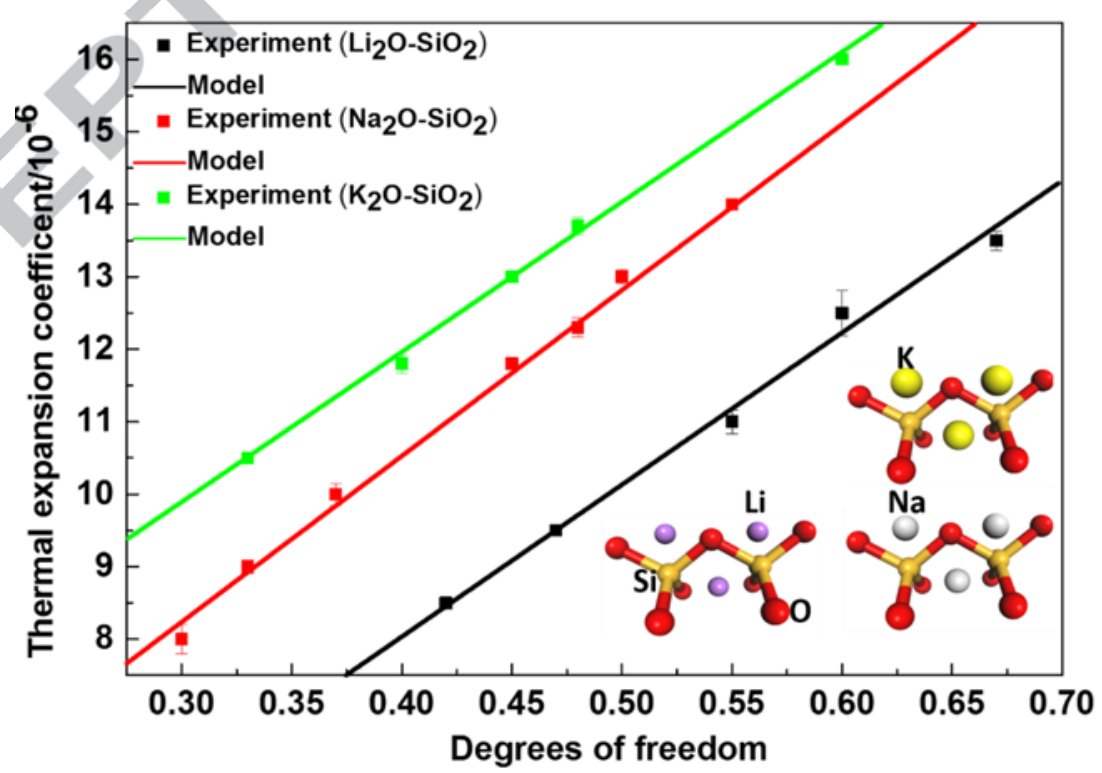

FIG. 2 Experimental TEC as functions of degrees of freedom of $x \mathrm{Na}_{2} \mathrm{O}-(1-x) \mathrm{SiO}_{2}$, $x \mathrm{~K}_{2} \mathrm{O}-(1-x) \mathrm{SiO}_{2}$, and $x \mathrm{Li}_{2} \mathrm{O}-(1-x) \mathrm{SiO}_{2}$ glasses. The square dots represent experimental results, the solid lines represent the predictions of the model. The inset shows the structures of the $\mathrm{Li}_{2} \mathrm{O}-\mathrm{SiO}_{2}, \mathrm{Na}_{2} \mathrm{O}-\mathrm{SiO}_{2}$, and $\mathrm{K}_{2} \mathrm{O}-\mathrm{SiO}_{2}$ glasses. 


\section{Calculation of Thermal Expansion Coefficient of Glasses Based on}

\section{Topological Constraint Theory}

The derived equation allows for the computation of TCT of glasses from degree of freedom. As presented in this paper, our approach has two advantages: it is empirical parameters-free compared with the early theoretical calculations, and this is a much more time and cost efficient method compared with molecular dynamic simulations.

The constraint strength index introduced in the paper for calculation of degree of freedom of alkali silicate glasses is already verified to overcome the limitations of TCT, which ignored the influence of glass modifiers in early investigations. Results showed that the calculated values are favored in the experimental results and the strong linear relationship were displayed. It is believed that this work established a configuration-based methodology to calculate the thermal expansion coefficient of the materials, like glasses, which lack periodic order. 